\title{
Early Dual Antiplatelet Therapy in Patients with Acute Minor Stroke Treated with Intravenous Thrombolysis
}

\author{
Marina Mannino Salvatore Cottone \\ Department of Neurology, AOOR Villa Sofia-V. Cervello, Palermo, Italy
}

\author{
Dear Editor, \\ We read the findings of Zhao et al. [1] with great inter-
} est.

Recently, 2 independent, multicenter, randomized, double-blind, placebo-controlled trials (CHANCE and POINT) have established the efficacy of short-term dual antiplatelet therapy (DAPT) to prevent recurrent ischemic stroke in patients with minor stroke or high-risk TIA[2,3]. Thus, starting DAPT (aspirin and clopidogrel) within $24 \mathrm{~h}$ after symptom onset is recommended by the most recent AHA guidelines [4] in patients presenting with minor noncardioembolic ischemic stroke (National Institutes of Health Stroke Scale [NIHSS] score $\leq 3$ ) who did not receive intravenous alteplase. (Patients who are candidates for thrombolysis were not represented in these trials, so results cannot be generalized to this group.)

Zhao et al. [1] treated 102 patients with aspirin and clopidogrel after intravenous thrombolysis for acute minor ischemic stroke and compared them with 105 patients treated with aspirin. Functional outcome was better in the dual antiplatelet group, without significant difference in terms of symptomatic intracerebral hemorrhage or mortality.

However, in this study, minor stroke was defined as a score of $\leq 5$ points on the National Institutes of Health

\footnotetext{
karger@karger.com

(c) 2020 S. Karger AG, Basel

www.karger.com/ene

Karger"
}

Stroke Scale (NIHSS). About one-third of these patients harbor a large vessel occlusion [5] and potentially develop a medium to large infarction with increased risk of hemorrhagic conversion. Thus, it would be useful to know how many patients in the study had an ischemic lesion on follow-up imaging and the size of the lesions.

Further, the half-life of alteplase is nearly $4 \mathrm{~min}$, but its effect on the coagulation system persists much longer. The fibrinolytic activity of alteplase is associated with consumptive coagulopathy with hypofibrinogenemia that may last $\geq 24 \mathrm{~h}$ after completion of the alteplase infusion. Early hypofibrinogenemia (fibrinogen level $<200$ $\mathrm{mg} / \mathrm{dL}$ at $2 \mathrm{~h}$ after alteplase infusion) occurs in about $20 \%$ of the patients after alteplase infusion and is associated with a substantially increased risk of symptomatic intracerebral hemorrhage [6].

In patients with minor stroke treated with intravenous thrombolysis, before starting early DAPT, it would be appropriate to consider the infarct size and exclude alteplase-related coagulopathy.

\section{Disclosure Statement}

The authors have no conflicts of interest to declare. 


\section{Funding Sources}

The authors did not receive any funding.

\section{Author Contributions}

M.M. contributed to the conception and drafting of the manuscript. S.C. contributed to critical revision and supervision.

\section{References}

1 Zhao G, Lin F, Wang Z, Shao X, Gong Y, Zhang S, et al. Dual antiplatelet therapy after intravenous thrombolysis for acute minor ischemic stroke. Eur Neurol. 2020;8:1-6.

2 Wang Y, Wang Y, Zhao X, Liu L, Wang D, Wang $\mathrm{C}$, et al. Clopidogrel with aspirin in acute minor stroke or transient ischemic attack. N Engl J Med. 2013;369:11-9.

3 Johnston SC, Easton JD, Farrant M, Barsan W, Conwit RA, Elm JJ, et al. Clopidogrel and aspirin in acute ischemic stroke and high-risk TIA. N Engl J Med. 2018;379:215-25.
4 Powers WJ, Rabinstein AA, Ackerson T, Adeoye OM, Bambakidis NC, Becker K, et al. Guidelines for the early management of patients with acute ischemic stroke: 2019 update to the 2018 guidelines for the early management of acute ischemic stroke: a guideline for healthcare professionals from the American heart association/American stroke association. Stroke. 2019;50:e344-418.
5 Griessenauer CJ, Medin C, Maingard J, Chandra RV, Ng W, Brooks DM, et al. Endovascular mechanical thrombectomy in large-vessel occlusion ischemic stroke presenting with Low National Institutes of Health Stroke Scale: systematic review and meta-analysis. World Neurosurg. 2018;110:263-9.

6 Vandelli L, Marietta M, Gambini M, Cavazzuti M, Trenti T, Cenci MA, et al. Fibrinogen decrease after intravenous thrombolysis in ischemic stroke patients is a risk factor for intracerebral hemorrhage. J Stroke Cerebrovasc Dis. 2015;24:394-400. 